Bull. Korean Math. Soc. 49 (2012), No. 6, pp. 1223-1240

http://dx.doi.org/10.4134/BKMS.2012.49.6.1223

\title{
A NOTE ON QUASI-PERIODIC PERTURBATIONS OF ELLIPTIC EQUILIBRIUM POINTS
}

\author{
HOUYu ZHAO
}

Abstract. The system

$$
\dot{x}=(A+\varepsilon Q(t, \varepsilon)) x+\varepsilon g(t, \varepsilon)+h(x, t, \varepsilon),
$$

where $A$ is elliptic whose eigenvalues are not necessarily simple and $h$ is $\mathcal{O}\left(x^{2}\right)$. It is proved that, under suitable hypothesis of analyticity, for most values of the frequencies, the system is reducible.

\section{Introduction and main result}

Before stating our questions, we first give some definitions and notations.

Definition 1.1. A function $f: \mathbb{R} \rightarrow \mathbb{R}$ is called real analytic quasi-periodic with the frequencies $\omega=\left(\omega_{1}, \omega_{2}, \ldots, \omega_{r}\right)$ if it can be represented as a Fourier series of the type

$$
f(t)=\sum_{k} f_{k} e^{\mathrm{i}\langle k, \omega\rangle t},
$$

where $k=\left(k_{1}, \ldots, k_{r}\right),\langle k, \omega\rangle=\sum k_{j} \omega_{j} \neq 0$ if $k \neq 0$, the coefficients $f_{k}$ decay exponentially with $|k|=\left|k_{1}\right|+\cdots+\left|k_{r}\right|$.

We denote by $Q(\omega)$ the set of real analytic quasi-periodic functions with the frequencies $\omega$.

From the above definition, we see that the function $F: \vartheta=\left(\vartheta_{1}, \ldots, \vartheta_{r}\right) \in$ $\mathbb{R}^{r} \rightarrow \mathbb{R}$ defined by

$$
F(\vartheta)=\sum_{k} f_{k} e^{\mathrm{i}\langle k, \vartheta\rangle}
$$

is $2 \pi$-periodic in each variable and bounded in a complex neighborhood of $\mathbb{R}^{r}:\left|\operatorname{Im} \vartheta_{j}\right| \leq \rho$ for some $\rho>0$. This function is called a shell function of $f$.

Received September 28, 2010; Revised December 09, 2011.

2010 Mathematics Subject Classification. 37C55, 34A30, 34C20.

Key words and phrases. quasi-periodic perturbations, elliptic points, quasi-periodic solutions, nonresonant condition, small divisors, quasi-periodic Floquet theorem, KAM theory.

This work was partially supported by the National Natural Science Foundation of China (Grant No. 10871117, 11171185), SDNSF (Grant No. ZR2010AM013) and Natural Science Foundation of Chongqing Normal University (Grant No. 12XLB003). 
Definition 1.2. Let $v(t)$ be a quasi-periodic function with rationally independent frequencies $\omega_{1}, \ldots, \omega_{r}$ and shell function $V$, satisfying $v(t)=V\left(\omega_{1} t, \ldots\right.$, $\left.\omega_{r} t\right)$. Then the limit

$$
\lim _{T \rightarrow \infty} \frac{1}{T} \int_{0}^{T} v(t) d t
$$

is called the time average of $v$, coincides with the space average

$$
v_{0}=\frac{1}{(2 \pi)^{r}} \int_{\mathbb{T}^{r}} V(\vartheta) d \vartheta
$$

and we denote the common value by $\bar{v}$ as well.

Definition 1.3. Let $Q_{\rho}(\omega) \subset Q(\omega)$ be the set of real analytic functions $f$ such that the corresponding shell functions $F$ are bounded on the subset $\Theta(\rho)=$ $\left\{\left(\vartheta_{1}, \ldots, \vartheta_{r}\right) \in \mathbb{C}^{r}:\left|\operatorname{Im} \vartheta_{j}\right| \leq \rho\right\}$, with the sup-norm

$$
\|f\|_{\rho}=\sup _{\vartheta \in \Theta(\rho)}\left|\sum_{k} f_{k} e^{\mathrm{i}\langle k, \vartheta\rangle}\right|=\sup _{\vartheta \in \Theta(\rho)}|F(\vartheta)| .
$$

By Cauchy's formula, it follows that

$$
\left|f_{k}\right| \leq\|f\|_{\rho} e^{-|k| \rho} \quad \text { and } \quad\left|f_{\vartheta}\right|_{\rho^{\prime}} \leq\left(\rho-\rho^{\prime}\right)^{-1}\|f\|_{\rho}
$$

for $f \in Q_{\rho}(\omega)$ and $0<\rho^{\prime}<\rho$.

Since N. N. Bogoljubov et al. [1] in 1960's proved reducibility of non-autonomous finite-dimensional linear systems to constant coefficient equations by KAM-technique, establishing the reducibility of finite-dimensional systems by means of the KAM tools has become an active field of research. Such results are also included in [11]. In this directions, we refer [2]-[16] and references therein for a detailed description. In particular, A. Jorba and C. Simó [9] investigated the reducibility of the quasi-periodic ordinary differential equation

$$
\dot{x}=(A+\varepsilon Q(t, \varepsilon)) x+\varepsilon g(t, \varepsilon)+h(x, t, \varepsilon),
$$

where $A$ is an elliptic constant square matrix with order $d$ (that is, all the eigenvalues are purely imaginary and nonzero), $Q$ is a square matrix with order $d, h$ is a vector function of second order in $x$, and $Q, g, h$ are quasi-periodic in time $t$ with frequency vector $\omega=\left(\omega_{1}, \omega_{2} \ldots, \omega_{r}\right)$. Such equation (1.3) is an autonomous differential equation under quasi-periodic time-dependent perturbations near an elliptic equilibrium point. More precisely, under suitable conditions of analyticity, nonresonance and nondegeneracy with respect to $\varepsilon$, A. Jorba and C. Simó [9] proved that the system (1.3) is reducible for $\varepsilon$ in some Cantorian set $\mathcal{E} \subset\left(0, \varepsilon_{0}\right)$ with $\varepsilon_{0}$ sufficiently small, provided that

(1) the eigenvalues $\lambda_{1}, \ldots, \lambda_{d}$ of $A$ are different;

(2) the eigenvalues $\lambda_{1}^{0}(\varepsilon), \ldots, \lambda_{d}^{0}(\varepsilon)$ of $\underline{A}=A+\varepsilon \bar{Q}(\varepsilon)+\overline{D_{x} h(\underline{x}(t, \varepsilon), t, \varepsilon)}$ satisfy

$$
0<2 \delta\left|\varepsilon_{1}-\varepsilon_{2}\right|<\left|\lambda_{i}^{0}\left(\varepsilon_{1}\right)-\lambda_{j}^{0}\left(\varepsilon_{1}\right)-\lambda_{i}^{0}\left(\varepsilon_{2}\right)+\lambda_{j}^{0}\left(\varepsilon_{2}\right)\right|<\frac{\bar{\delta}}{2}\left|\varepsilon_{1}-\varepsilon_{2}\right|,
$$




$$
0<2 \delta\left|\varepsilon_{1}-\varepsilon_{2}\right|<\left|\lambda_{k}^{0}\left(\varepsilon_{1}\right)-\lambda_{k}^{0}\left(\varepsilon_{2}\right)\right|<\frac{\bar{\delta}}{2}\left|\varepsilon_{1}-\varepsilon_{2}\right|
$$

for all $i, j$, and $k$ satisfying $1 \leq i<j \leq d$ and $1 \leq k \leq d$, and provided that $\left|\varepsilon_{1}\right|$ and $\left|\varepsilon_{2}\right|$ are less than some small value $\varepsilon_{0}$. Here $\underline{x}(t)$ is the unique analytical quasi-periodic solution of $\dot{x}=A x+\varepsilon g(t, \varepsilon)$.

Naturally, we should ask that whether or not (1.3) is reducible when conditions (1) or (2) is not satisfied. In this paper, we will give an answer to this question.

Throughout this paper, we always assume that the following hypothesis is satisfied:

(H) $Q(t), g(t)$, and $h(t)$ are in $Q_{\rho}(\omega)$ and $\omega \in D_{\gamma}$ :

$$
\begin{aligned}
D_{\gamma}:= & \left\{\omega \in \mathbb{R}^{r}:|| \sqrt{-1}(k, \omega) E_{d}-\left.A\right|_{e} \mid>\frac{\gamma}{|k|^{\tau}},\right. \\
& \left.|| \sqrt{-1}(k, \omega) E_{d^{2}}-E_{d} \otimes A+\left.A^{\top} \otimes E_{d}\right|_{e} \mid>\frac{\gamma}{|k|^{\tau}}\right\},
\end{aligned}
$$

where $k \in \mathbb{Z}^{r} \backslash\{0\}, 1 \leq i, j \leq d, \gamma>0, \tau \geq r-1$ and $|\cdot|_{e}$ denotes the determinant of a matrix.

If $x \in \mathbb{R}^{n}$, we denote by $\|x\|$ the sup norm of $x$. If $A$ is a matrix, $\|A\|$ denotes the corresponding sup-norm, and for a matrix-valued function $Q(t)$, define

$$
\|Q\|^{U}=\sup _{t \in U}\|Q(t)\|
$$

where $\|\cdot\|$ is the sup-norm of the matrix.

In the present paper, we will follow the techniques developed in [9] and [16] to prove the following result.

Theorem 1.1. Assume that $(\mathrm{H})$ is satisfied. Let $\Omega_{0} \subset \mathbb{R}^{r}$ be a compact set with positive Lebesgue measure. Consider the system (1.3) and assume that

(A1) $\operatorname{det} A \neq 0$, and $Q(t, \varepsilon), g(t, \varepsilon)$ and $h(x, t, \varepsilon)$ are in $Q_{\rho}(\omega)$ with $\omega \in \Omega_{0}$.

(A2) $h(x, t, \varepsilon)$ is analytic with respect to $x$ on the ball $B_{\kappa}(0)$ centered in the origin with radius $\kappa, h(0, t, \varepsilon)=0$ and $\left\|D_{x x} h(x, t, \varepsilon)\right\| \leq K$ with $\|x\| \leq \kappa$ and $K$ positive constant. Then for a sufficiently small positive constant $\gamma$, there exist a subset $\Omega \subset \Omega_{0}$ with Meas $\left(\Omega_{0} \backslash \Omega\right)=\operatorname{Meas}\left(\Omega_{0}\right)\left(1-O\left(\gamma^{\frac{1}{d^{2}}}\right)\right)$ and a sufficiently small constant $\epsilon(\rho, \gamma)>0$ such that for any $\varepsilon \in(0, \epsilon)$, there is an analytic quasi-periodic transformation $y=\Psi(t) x$ with the basic frequencies $\omega$ such that the system (1.3) is transformed into

$$
\dot{y}=A_{\infty}(\varepsilon) y+h_{\infty}(y, t, \varepsilon),
$$

where $A_{\infty}$ is a constant matrix and $h_{\infty}(y, t, \varepsilon)$ is of second order in $y$.

Corollary 1. Under the hypothesis of Theorem 1.1, for any $\varepsilon \in(0, \epsilon),(1.3)$ has a quasi-periodic solution $x_{\varepsilon}(t)$ with basic frequencies $\omega$ such that

$$
\lim _{\substack{\varepsilon \rightarrow 0 \\ \varepsilon \in E}}\left\|x_{\varepsilon}\right\|=0 \text {. }
$$




\section{Preliminary lemmas}

Lemma 2.1. Let us consider the equation

$$
\dot{x}=A x+\varepsilon g(t),
$$

where $A$ is a $d \times d$ matrix with $\operatorname{det} A \neq 0$ and $g(t) \in Q_{\rho_{1}}(\omega)$. If $0<\rho_{2}<\rho_{1}$ and $\omega \in D_{\gamma}$, then the equation (2.1) has a unique quasi-periodic solution $x(t) \in$ $Q_{\rho_{2}}(\omega)$ satisfying

$$
\|x\|_{\rho_{2}} \leq \varepsilon L_{1}\|g\|_{\rho_{1}},
$$

where $L_{1}=\left\|A^{-1}\right\|+\frac{C}{\gamma}\left(\frac{\tau}{e}\right)^{\tau} \frac{(1+e)^{r}}{\left(\frac{\rho_{1}-\rho_{2}}{2}\right)^{\tau+r}}$.

Proof. We write

$$
x(t)=\sum_{k \in \mathbb{Z}^{r}} x_{k} e^{\sqrt{-1}(k, \omega) t}, \quad g(t)=\sum_{k \in \mathbb{Z}^{r}} g_{k} e^{\sqrt{-1}(k, \omega) t},
$$

where $x_{k}$ and $g_{k}$ are vectors. From $\dot{x}=A x+\varepsilon g(t)$ we get

$$
\left(\sqrt{-1}(k, \omega) E_{d}-A\right) x_{k}=\varepsilon g_{k},
$$

where $E_{d}$ is a $d$ dimension unit matrix.

When $k=0$, because $\operatorname{det} A \neq 0$, we get

$$
\left|x_{0}\right| \leq \varepsilon\left\|A^{-1}||_{\rho_{2}}\left|g_{0}\right| \leq \varepsilon\right\| A^{-1}\left\|_{\rho_{1}}\right\| g \|_{\rho_{1}} .
$$

When $k \neq 0$, by $(\mathrm{H})$ and (1.2), the equation (2.2) is solvable for $\omega \in D_{\gamma}$, and

$$
\left|x_{k}\right| \leq \varepsilon\left\|\left(\sqrt{-1}(k, \omega) E_{d}-A\right)^{-1}\right\|_{\rho_{2}}\left|g_{k}\right| \leq \varepsilon C \frac{|k|^{\tau}}{\gamma}\|g\|_{\rho_{1}} e^{-\rho_{1}|k|} .
$$

Further, we have

$$
\begin{aligned}
\|x\|_{\rho_{2}} & \leq \varepsilon\left\|A^{-1}\right\|_{\rho_{1}}\|g\|_{\rho_{1}}+\sum_{k \neq 0} \varepsilon C \frac{|k|^{\tau}}{\gamma}\|g\|_{\rho_{1}} e^{-\left(\rho_{1}-\rho_{2}\right)|k|} \\
& \leq \varepsilon\left[\left\|A^{-1}\right\|+\frac{C}{\gamma}\left(\frac{\tau}{e}\right)^{\tau} \frac{(1+e)^{r}}{\left(\frac{\rho_{1}-\rho_{2}}{2}\right)^{\tau+r}}\right]\|g\|_{\rho_{1}} \\
& =\varepsilon L_{1}\|g\|_{\rho_{1}},
\end{aligned}
$$

where the second inequality follows from Lemma 6.1 .

Lemma 2.2. Consider the equation

$$
\dot{P}=A P-P A+Q^{*},
$$

where $A$ is a $d \times d$ matrix and $Q^{*}(t)=\sum_{0<|k| \leq M} Q_{k} e^{\sqrt{-1}(k, \omega) t} \in Q_{\rho_{1}}(\omega)$. Let $0<\rho_{2}<\rho_{1}$ and $\omega \in D_{\gamma}$. Then the equation (2.5) has a unique quasi-periodic solution $P(t) \in Q_{\rho_{2}}(\omega)$ satisfying

$$
\|P\|_{\rho_{2}} \leq L_{2}\|Q\|_{\rho_{1}},
$$

where $Q=\sum_{k \in \mathbb{Z}^{r}} Q_{k} e^{\sqrt{-1}(k, \omega) t}, L_{2}=\frac{C}{\gamma}\left(\frac{\tau}{e}\right)^{\tau} \frac{(1+e)^{r}}{\left(\frac{\rho_{1}-\rho_{2}}{2}\right)^{\tau+r}}$. 
Proof. Let

$$
P(t)=\sum_{0<|k| \leq M} P_{k} e^{\sqrt{-1}(k, \omega) t} .
$$

Then it is easy to see that

$$
\left(\sqrt{-1}(k, \omega) E_{d}-A\right) P_{k}+P_{k} A=Q_{k} .
$$

By $(\mathrm{H}),(1.2)$ and Lemma 6.1, we have

$$
\begin{aligned}
\left|P_{k}\right| & \leq\left\|\left(\sqrt{-1}(k, \omega) E_{d^{2}}-E_{d} \otimes A+A^{\top} \otimes E_{d}\right)^{-1}\right\|_{\rho_{2}}\left|Q_{k}\right| \\
& \leq C \frac{|k|^{\tau}}{\gamma}\|Q\|_{\rho_{1}} e^{-\rho_{1}|k|}
\end{aligned}
$$

and

$$
\begin{aligned}
\|P\|_{\rho_{2}} & \leq \sum_{0<|k| \leq M} C \frac{|k|^{\tau}}{\gamma}\|Q\|_{\rho_{1}} e^{-\rho_{1}|k|} e^{\rho_{2}|k|} \\
& \leq \sum_{k \in \mathbb{Z}^{r}} C \frac{|k|^{\tau}}{\gamma}\|Q\|_{\rho_{1}} e^{-\left(\rho_{1}-\rho_{2}\right)|k|} \\
& \leq \frac{C}{\gamma}\left(\frac{\tau}{e}\right)^{\tau} \frac{(1+e)^{r}}{\left(\frac{\rho_{1}-\rho_{2}}{2}\right)^{\tau+r}}\|Q\|_{\rho_{1}} .
\end{aligned}
$$

Lemma 2.3. Let us consider

$$
\dot{x}=(A+\varepsilon Q(t)) x+\varepsilon g(t)+h(x, t),
$$

where $Q(t), g(t), h(x, t) \in Q_{\rho_{1}}(\omega)$ and $0<\rho_{2}<\rho_{1}$. Also, we assume that $h(x, t)$ is analytic with respect to $x$ on the ball $B_{\kappa}(0)$ and satisfies $\left\|D_{x, x} h(x, t)\right\|_{\rho_{1}} \leq$ $K, \forall x \in B_{\kappa}(0)$. Then for a solution $\underline{x}(t) \in Q_{\rho_{2}}(\omega)$ of the equation $(2.1)$, the change of variables $x=y+\underline{x}(t)$ transforms the initial equation (2.9) into

$$
\dot{y}=\left(\bar{A}+\varepsilon \widetilde{Q}_{1}(t)\right) y+\varepsilon_{1} g_{1}(t)+h_{1}(y, t),
$$

where $\varepsilon_{1}=\varepsilon^{1+\iota}$ and $\widetilde{Q}_{1}$ has zero average and the following bounds hold for $\omega \in D_{\gamma}$ :

1. $\left\|\widetilde{Q}_{1}\right\|_{\rho_{2}} \leq 2\|Q\|_{\rho_{1}}+2 K L_{1}\|g\|_{\rho_{1}}$, where $L_{1}$ was defined in Lemma 2.1.

2. $\left\|g_{1}\right\|_{\rho_{2}} \leq\left(\frac{K L_{1}^{2}\left(\|g\|_{\rho_{1}}\right)^{2}}{2}+L_{1}\|Q\|\left\|_{\rho_{1}}\right\| g \|_{\rho_{1}}\right) \varepsilon^{1-\iota}$.

3. $\|\bar{A}\| \leq\|A\|+\varepsilon\left(\|Q\|_{\rho_{1}}+K L_{1}\|g\|_{\rho_{1}}\right)$.

4. $\left\|D_{y y} h_{1}\right\|_{\rho_{2}} \leq K$.

5. $\|\underline{x}\|_{\rho_{2}} \leq \varepsilon L_{1}\|g\|_{\rho_{1}}$.

Here $y \in B_{\kappa_{1}}(0), \kappa_{1}=\kappa-\|\underline{x}\|_{\rho_{2}}$, and $\varepsilon$ is small enough.

Proof. Let $\underline{x}$ be such that $\underline{\dot{x}}=A \underline{x}+\varepsilon g$. In Lemma 2.1, we have

$$
\|\underline{x}\|_{\rho_{2}} \leq \varepsilon L_{1}\|g\|_{\rho_{1}} .
$$


By the change of variables $x=y+\underline{x}(t)$, we can obtain

$$
\dot{y}=\left(A+\varepsilon Q_{1}(t)\right) y+\varepsilon_{1} g_{1}(t)+h_{1}(y, t),
$$

where $Q_{1}=Q+\frac{1}{\varepsilon} D_{x} h(\underline{x}(t), t), g_{1}=\frac{1}{\varepsilon^{1+\iota}} h(\underline{x}(t), t)+\frac{1}{\varepsilon^{\iota}} Q \underline{x}(t)(\varepsilon \neq 0)$, and $h_{1}(y, t)=h(\underline{x}(t)+y, t)-h(\underline{x}(t), t)-D_{x} h(\underline{x}(t), t) y$. Like [9], the terms of this equation must be bounded. First, by Lemma 6.4 , we have

$$
\left\|Q_{1}\right\|_{\rho_{2}} \leq\|Q\|_{\rho_{2}}+\frac{1}{\varepsilon} K\|\underline{x}\|_{\rho_{2}} \leq\|Q\|_{\rho_{1}}+K L_{1}\|g\|_{\rho_{1}} .
$$

Then we bound $\left\|g_{1}\right\|_{\rho_{2}}$, also from Lemma 6.4 , we get

$$
\begin{aligned}
\left\|g_{1}\right\|_{\rho_{2}} & \leq \frac{K}{2} \frac{1}{\varepsilon^{1+\iota}}\left(\|\underline{x}\|_{\rho_{2}}\right)^{2}+\frac{1}{\varepsilon^{\iota}}\|Q\|_{\rho_{1}}\|\underline{x}\|_{\rho_{2}} \\
& \leq\left(\frac{K L_{1}^{2}\left(\|g\|_{\rho_{1}}\right)^{2}}{2}+L_{1}\|Q\|\left\|_{\rho_{1}}\right\| g \|_{\rho_{1}}\right) \varepsilon^{1-\iota} .
\end{aligned}
$$

The third one is $D_{y y} h_{1}(y, t)$,

$$
\left\|D_{y y} h_{1}\right\|_{\rho_{2}}=\left\|D_{x x} h(\underline{x}(t)+y, t)\right\| \leq K .
$$

So we must require that $y \in B_{\kappa_{1}}(0)$, where $\kappa_{1}=\kappa-\|\underline{x}\|_{\rho_{2}}$. We define $Q_{1}(t)=$ $\bar{Q}_{1}+\widetilde{Q}_{1}(t), \bar{A}=A+\varepsilon \bar{Q}_{1}$, we obtain

$$
\dot{y}=\left(\bar{A}+\varepsilon \widetilde{Q}_{1}(t)\right) y+\varepsilon_{1} g_{1}(t)+h_{1}(y, t) .
$$

At last,

$$
\begin{aligned}
\|\bar{A}\| & \leq\|A\|+\varepsilon\left\|\bar{Q}_{1}\right\|_{\rho_{2}} \\
& \leq\|A\|+\varepsilon\left\|Q_{1}\right\|_{\rho_{2}} \\
& \leq\|A\|+\varepsilon\left(\|Q\|_{\rho_{1}}+K L_{1}\|g\|_{\rho_{1}}\right)
\end{aligned}
$$

and

$$
\left\|\widetilde{Q}_{1}\right\|_{\rho_{2}} \leq 2\left\|Q_{1}\right\|_{\rho_{2}} \leq 2\left(\|Q\|_{\rho_{1}}+K L_{1}\|g\|_{\rho_{1}}\right) .
$$

Lemma 2.4. Let us consider

$$
\dot{x}=(A+\varepsilon Q(t)) x+\varepsilon_{1} g(t)+h(x, t),
$$

where $Q(t), g(t), h(x, t) \in Q_{\rho_{1}}(\omega), 0<\rho_{2}<\rho_{1}$ and $Q$ has zero average. Also, we assume that $h(x, t)$ is analytic with respect to $x$ on the ball $B_{\kappa}(0)$ and satisfies $\left\|D_{x x} h(x, t)\right\|_{\rho_{1}}^{\mathcal{O}_{1}} \leq K, \forall x \in B_{\kappa}(0)$. Then the change of variables $x=$ $(E+\varepsilon P(t)) y$ with $P(t) \in Q_{\rho_{2}}(\omega)$ transforms the initial equation (2.10) into

$$
\dot{y}=\left(\bar{A}+\varepsilon_{1} \widetilde{Q}_{1}(t)\right) y+\varepsilon_{1} g_{1}(t)+h_{1}(y, t),
$$

where $\widetilde{Q}_{1}$ has zero average, $E$ is the identity $d \times d$ matrix and the following bounds hold for $\omega \in D_{\gamma}$ :

1. $\left\|\widetilde{Q}_{1}\right\|_{\rho_{2}} \leq 2 \varepsilon^{1-\iota} \frac{\left(L_{2}^{\prime}+\|P\|_{\rho_{2}}\right)}{1-\left.\varepsilon\|P\|\right|_{\rho_{2}}}\|Q\|_{\rho_{1}}$, where $\|P\|_{\rho_{2}} \leq L_{2}\|Q\|_{\rho_{1}}$ and $L_{2}$ was defined in Lemma 2.2.
2. $\left\|g_{1}\right\|_{\rho_{2}} \leq \frac{1}{1-\varepsilon\|P\| \rho_{2}}\|g\|_{\rho_{1}}$.
3. $\|\bar{A}\| \leq\|A\|+\varepsilon^{2} \frac{\left(L_{2}^{\prime}+\|P\|_{\rho_{2}}\right)}{1-\varepsilon\|P\| \|_{\rho_{2}}}\|Q\|_{\rho_{1}}$. 
4. $\left\|D_{y y} h_{1}\right\|_{\rho_{2}} \leq K \frac{\left(1+\varepsilon\|P\| \|_{\rho_{2}}\right)^{2}}{1-\varepsilon\|P\| \|_{\rho_{2}}}$.

Here $y \in B_{\kappa_{2}}(0)$, where $\kappa_{2}=\frac{\kappa}{1+\varepsilon\|P\| \|_{\rho_{2}}}$, and $\varepsilon$ is small enough.

Proof. By the change of variables $x=(E+\varepsilon P) y$, we can obtain

$\dot{y}=(E+\varepsilon P)^{-1}\left(A+\varepsilon\left(Q^{*}+A P-\dot{P}\right)+\left(\varepsilon Q^{* *}+\varepsilon^{2} Q P\right)\right) y+\varepsilon_{1} g_{1}(t)+h_{1}(y, t)$,

where

$$
\begin{aligned}
Q & =Q^{*}+Q^{* *}, \\
Q^{*}(t) & =\sum_{0<|k| \leq M} Q_{k} e^{\sqrt{-1}(k, \omega) t}, \\
Q^{* *}(t) & =\sum_{|k|>M} Q_{k} e^{\sqrt{-1}(k, \omega) t}, \\
g_{1} & =(E+\varepsilon P)^{-1} g, \text { and } \\
h_{1}(y, t) & =(E+\varepsilon P)^{-1} h((E+\varepsilon P) y, t) .
\end{aligned}
$$

We would like to have

$$
(E+\varepsilon P)^{-1}\left(A+\varepsilon\left(Q^{*}+A P-\dot{P}\right)\right)=A,
$$

this implies that

$$
\dot{P}=A P-P A+Q^{*} .
$$

From Lemma 2.2 we have

$$
\|P\|_{\rho_{2}}<L_{2}\|Q\|_{\rho_{1}}
$$

We obtain the equation

$$
\dot{y}=\left(A+\varepsilon_{1} Q_{1}(t)\right) y+\varepsilon_{1} g_{1}(t)+h_{1}(y, t),
$$

where $\varepsilon_{1} Q_{1}=(E+\varepsilon P)^{-1}\left(\varepsilon Q^{* *}+\varepsilon^{2} Q P\right)$.

Now we bounded the terms of this equation. In fact

$$
\begin{aligned}
\left\|Q^{* *}\right\|_{\rho_{2}} & \leq \sum_{|k|>M}\left\|Q_{k}\right\|_{\rho_{2}} e^{\rho_{2}|k|} \\
& \leq\|Q\|_{\rho_{1}} \sum_{|k|>M} e^{-\left(\rho_{1}-\rho_{2}\right)|k|} \\
& =\|Q\|_{\rho_{1}} \sum_{|k|>0} e^{-\left(\rho_{1}-\rho_{2}\right)(|k|+M)} \\
& \leq \varepsilon\|Q\|_{\rho_{1}} \sum_{|k|>0} e^{-\left(\rho_{1}-\rho_{2}\right)|k|} \\
& \leq \varepsilon L_{2}^{\prime}\|Q\|_{\rho_{1}}
\end{aligned}
$$

then

$$
\left\|\varepsilon_{1} Q_{1}\right\|_{\rho_{2}} \leq \frac{1}{1-\varepsilon\|P\|_{\rho_{2}}}\left(\varepsilon\left\|Q^{* *}\right\|_{\rho_{2}}+\varepsilon^{2}\|Q\|_{\rho_{2}}\|P\|_{\rho_{2}}\right)
$$




$$
\leq \varepsilon^{2} \frac{\left(L_{2}^{\prime}+\|P\|_{\rho_{2}}\right)}{1-\varepsilon\|P\| \|_{\rho_{2}}}\|Q\|_{\rho_{1}}
$$

and

$$
\begin{gathered}
\left\|Q_{1}\right\|_{\rho_{2}} \leq \varepsilon^{1-\iota} \frac{\left(L_{2}^{\prime}+\|P\|_{\rho_{2}}\right)}{1-\varepsilon\|P\| \|_{\rho_{2}}}\|Q\|_{\rho_{1}}, \\
\left\|g_{1}\right\|_{\rho_{2}} \leq \frac{1}{1-\varepsilon\|P\|_{\rho_{2}}}\|g\|_{\rho_{1}}, \\
\left\|D_{y y} h_{1}\right\|_{\rho_{2}} \leq \frac{K}{1-\varepsilon\|P\| \|_{\rho_{2}}}\left(\|E+\varepsilon P\|_{\rho_{2}}\right)^{2} \leq K \frac{\left(1+\varepsilon\|P\| \|_{\rho_{2}}\right)^{2}}{1-\varepsilon\|P\|_{\rho_{2}}},
\end{gathered}
$$

in this we require $y \in B_{\kappa_{2}}(0)$, where $\kappa_{2}=\frac{\kappa}{1+\varepsilon|| P \|\left.\right|_{\rho_{2}}}$, and $\varepsilon$ is small enough. Let $Q_{1}(t)=\bar{Q}_{1}+\widetilde{Q}_{1}(t), \bar{A}=A+\varepsilon_{1} \bar{Q}_{1}$. Then we obtain

$$
\dot{y}=\left(\bar{A}+\varepsilon_{1} \widetilde{Q}_{1}(t)\right) y+\varepsilon_{1} g_{1}(t)+h_{1}(y, t) .
$$

At last,

$$
\begin{gathered}
\|\bar{A}\| \leq\|A\|+\varepsilon_{1}\left\|Q_{1}\right\|_{\rho_{2}} \leq\|A\|+\varepsilon^{2} \frac{\left(L_{2}^{\prime}+\|P\|_{\rho_{2}}\right)}{1-\varepsilon\|P\|_{\rho_{2}}}\|Q\|_{\rho_{1}}, \\
\left\|\widetilde{Q}_{1}\right\|_{\rho_{2}} \leq 2\left\|Q_{1}\right\|_{\rho_{2}} \leq 2 \varepsilon^{1-\iota} \frac{\left(L_{2}^{\prime}+\|P\| \|_{\rho_{2}}\right)}{1-\varepsilon\|P\| \|_{\rho_{2}}}\|Q\|_{\rho_{1}} .
\end{gathered}
$$

\section{Iterative lemma}

We will invoke the KAM iterative technique to prove Theorem 1.1. To that end, for given $\varepsilon>0, \rho>0$ and $r>0$, we first introduce some iterative sequences.

(i) $\varepsilon_{0}=\varepsilon, \quad \varepsilon_{n}=\varepsilon^{(1+\iota)^{n-1}}, \quad \iota=\frac{1}{4}, \quad n=1,2, \ldots$;

(ii) $\rho_{0}=\rho, \quad \rho_{n}=\rho_{0}-\frac{\rho_{0}}{2} \frac{\left(1+2^{-2}+\cdots+n^{-2}\right)}{\sum_{j=1}^{\infty} j^{-2}}, \quad n=0,1,2, \ldots$;

(iii) $q_{n}=\varepsilon_{n+1}^{\frac{1}{4 d^{2}}}, \quad n=0,1,2, \ldots$, where $d$ is the dimensional number of system (1.3);

(iv) $\Theta_{n}=\Theta\left(\rho_{n}\right)=\left\{\vartheta=\left(\vartheta_{1}, \vartheta_{2}, \ldots, \vartheta_{r}\right) \in \mathbb{C}^{r} / 2 \pi \mathbb{Z}^{r}:|\operatorname{Im} \vartheta|<\rho_{n}\right\}, \quad n=$ $0,1,2, \ldots$;

(v) $\sigma_{n}=\rho_{0}-\frac{\rho_{0}}{2} \frac{\left(1+2^{-2}+\cdots+n^{-2}+\frac{(n+1)-2}{2}\right)}{\sum_{j=1}^{\infty} j^{-2}}, \quad \widetilde{q}_{n}=\frac{q_{n}+q_{n+1}}{2}$.

Let $\Pi_{0}=\Omega_{0}$ and define the sets

$$
\begin{gathered}
\mathcal{R}_{k}^{*}(n)=\left\{\omega \in \Pi_{n-1}:|| \sqrt{-1}(k, \omega) E_{d^{2}}-E_{d} \otimes A_{n}+\left.A_{n}^{\top} \otimes E_{d}\right|_{e} \mid<\frac{\gamma_{n}}{|k|^{\tau_{1}}}\right\}, \\
\mathcal{R}_{k}^{* *}(n)=\left\{\omega \in \Pi_{n-1}:|| \sqrt{-1}(k, \omega) E_{d}-\left.A_{n}\right|_{e} \mid<\frac{\gamma_{n}}{|k|^{\tau_{1}}}\right\}, \\
\mathcal{R}_{k}(n)=\mathcal{R}_{k}^{*}(n) \bigcup \mathcal{R}_{k}^{* *}(n),
\end{gathered}
$$


where $\gamma_{n}=\frac{\gamma}{n^{2 d^{2}}}$ and $\tau_{1}=(r+1) d^{2}$ and

$$
\Pi_{n}=\Pi_{n-1} \backslash \bigcup_{0<|k|<M_{n}} \mathcal{R}_{k}(n),
$$

where $M_{n}=\frac{\left|\ln \varepsilon_{n}\right|}{\rho_{n-1}-\rho_{n}}$. Then $\Pi_{n}$ is the sequence of compact closed subsets of $\mathbb{R}_{+}^{r}$ with

$$
\Omega_{0}=\Pi_{0} \supset \Pi_{1} \cdots \supset \Pi_{n} \supset \cdots
$$

and from Lemma 5.2, we have

$$
\operatorname{Meas} \mathcal{R}_{k}(n) \leq \frac{2}{n^{2}} \frac{R \gamma^{\frac{1}{d^{2}}}}{|k|^{r+1}},
$$

and

$$
\begin{aligned}
\text { Meas } \bigcup_{0<|k|<M_{n}} \mathcal{R}_{k}(n) & \leq \sum_{0 \neq k \in \mathbb{Z}^{r}} \operatorname{Meas\mathcal {R}}_{k}(n) \\
& \leq \frac{2}{n^{2}} R \gamma^{\frac{1}{d^{2}}} \sum_{0 \neq k \in \mathbb{Z}^{r}} \frac{1}{|k|^{r+1}} \\
& \leq \frac{4 r}{n^{2}} R \gamma^{\frac{1}{d^{2}}} \sum_{m=1}^{\infty} m^{r-1} \frac{1}{m^{r+1}}=\frac{2 \pi^{2} r}{3 n^{2}} R \gamma^{\frac{1}{d^{2}}}
\end{aligned}
$$

where we use that $\#\left\{k \in \mathbb{Z}^{r} /|k|=m\right\} \leq 2 r m^{r-1}$.

Then, we can prove that

$$
\begin{aligned}
\operatorname{Meas} \Pi_{n} & =\operatorname{Meas}\left(\Pi_{0}-\bigcup_{i=1}^{n} \bigcup_{0<|k|<M_{i}} \mathcal{R}_{k}(i)\right) \\
& >\left(1-\frac{2 \pi^{2}}{3} r R\left(\sum_{i=1}^{n} \frac{1}{i^{2}}\right) \gamma^{\frac{1}{d^{2}}}\right) \text { Meas } \Pi_{0},
\end{aligned}
$$

and

$$
\operatorname{Meas} \Pi_{\infty}=\left(1-O\left(\gamma^{\frac{1}{d^{2}}}\right)\right) \text { Meas } \Pi_{0} .
$$

We let that $\mathcal{O}_{n}$ and $U_{n}$ are the complex $q_{n}$-neighborhood and $\widetilde{q}_{n}$-neighborhood of $\Pi_{n}$ respectively, and construct iteratively a series of equations of the form

$(E)_{n} \quad \dot{x}_{n}=\left(A_{n}(\omega)+\varepsilon_{n} Q_{n}(t, \omega)\right) x_{n}+\varepsilon_{n} g_{n}(t, \omega)+h_{n}\left(x_{n}, t, \omega\right)$,

and where the following conditions are satisfied:

$(\mathrm{H} 1)_{n} Q_{n}(t, \omega)$ and $g_{n}(t, \omega)$ are analytic in $\Theta_{n} \times \mathcal{O}_{n}$, and

$$
\max \left\{\left\|Q_{n}(t, \omega)\right\|^{\Theta_{n} \times \mathcal{O}_{n}},\left\|g_{n}\right\|^{\Theta_{n} \times \mathcal{O}_{n}}\right\} \leq 1 .
$$

$(\mathrm{H} 2)_{n} h_{n}\left(x_{n}, t, \varepsilon\right)$ is analytic with respect to $x_{n}$ on the ball $B_{\kappa_{n}}(0)$, where $\kappa_{n}$ is a sequence with $\kappa_{0}=\kappa$ and $\lim _{n \rightarrow \infty} \kappa_{n}>0$. 
Lemma 3.1 (Iterative lemma). Assume that $(\mathrm{H} 1)_{n}$ and $(\mathrm{H} 2)_{n}$ are satisfied. Then for $\varepsilon$ small sufficiently there is a transformation

$$
\Phi_{n}: \quad x_{n}=\left(E+\varepsilon_{n} P_{n}(t, \omega)\right) x_{n+1}+\underline{x_{n}},
$$

where $\underline{x_{n}}$ satisfies $\underline{\dot{x}_{n}}(t)=A_{n} \underline{x_{n}}(t)+\varepsilon_{n} g_{n}(t)$ and $P_{n}(t, \omega)$ is analytic in $\Theta_{n} \times \mathcal{O}_{n}$ and quasi-periodic with frequency $\omega$, such that $(E)_{n}$ is changed into $(E)_{n+1}$ and $(\mathrm{H} 1)_{n+1}$ and $(\mathrm{H} 2)_{n+1}$ are fulfilled.

Proof. Now we apply the change of variables $x_{n}(t)=y_{n}(t)+\underline{x_{n}}(t)$, where $\underline{x_{n}}$ satisfies $\underline{\dot{x}_{n}}(t)=A_{n} \underline{x_{n}}(t)+\varepsilon_{n} g_{n}(t)$, and Lemma 2.3 to $(E)_{n}$. Then we get

$$
\dot{y}_{n}=\left(\widehat{A}_{n}(\omega)+\varepsilon_{n} \widehat{Q}_{n}(t, \omega)\right) x_{n}+\varepsilon_{n+1} \widehat{g}_{n}(t, \omega)+\widehat{h}_{n}\left(x_{n}, t, \omega\right)
$$

where the analyticity strip reduce to $\sigma_{n}$, and

$$
\begin{aligned}
\widehat{A}_{n} & =A_{n}+\varepsilon_{n} \bar{Q}_{n}(t)+\overline{D_{x} h_{n}\left(\underline{x_{n}}(t), t\right)}, \\
\widehat{Q}_{n}(t) & =Q_{n}(t)-\bar{Q}_{n}(t)+\frac{1}{\varepsilon_{n}}\left(D_{x} h_{n}\left(\underline{x_{n}}(t), t\right)-\overline{D_{x} h_{n}\left(\underline{x_{n}}(t), t\right)}\right), \\
\widehat{g}_{n}(t) & =\varepsilon_{n}^{-(1+\iota)} h_{n}\left(\underline{x_{n}}(t), t\right)+\varepsilon_{n}^{-\iota} Q_{n}(t) \underline{x_{n}}(t), \\
\widehat{h}_{n}(t) & \left.=h_{n} \underline{x_{n}}(t)+y_{n}, t\right)-h_{n}\left(\underline{x_{n}}(t), t\right)-D_{x} h_{n}\left(\underline{x_{n}}(t), t\right) y_{n},
\end{aligned}
$$

where we omit the dependence on $\omega$ to simplify the notation.

Then we apply the change of variables $y_{n}(t)=\left(E+\varepsilon_{n} P_{n}(t)\right) x_{n+1}(t)$ and Lemma 2.4 to (3.5), we have

$\dot{x}_{n+1}=\left(A_{n+1}(\omega)+\varepsilon_{n+1} Q_{n+1}(t, \omega)\right) x_{n+1}+\varepsilon_{n+1} g_{n+1}(t, \omega)+h_{n+1}\left(x_{n+1}, t, \omega\right)$.

Now the analyticity strip has been reduced to $\rho_{n+1}$, and

$$
\begin{aligned}
A_{n+1} & =\widehat{A}_{n}+\overline{\left(E+\varepsilon_{n} P_{n}(t)\right)^{-1}}\left(\varepsilon_{n} \overline{\widehat{Q}_{n}^{* *}}(t)+\varepsilon_{n}^{2} \overline{\widehat{Q}}_{n}(t) \bar{P}_{n}(t)\right) \\
= & A_{n}+\varepsilon_{n} \bar{Q}_{n}(t)+\overline{D_{x} h_{n}\left(\underline{x_{n}}(t), t\right)} \\
Q_{n+1}(t)= & \varepsilon_{n+1}^{-1}\left(E+\varepsilon_{n} P_{n}(t)\right)^{-1}\left(\varepsilon_{n} \widehat{Q}_{n}^{* *}(t)+\varepsilon_{n}^{2} \widehat{Q}_{n}(t) P_{n}(t)\right) \\
& -\varepsilon_{n+1}^{-1} \overline{\left(E+\varepsilon_{n} P_{n}(t)\right)^{-1}}\left(\varepsilon_{n} \overline{\widehat{Q}_{n}^{* *}}(t)+\varepsilon_{n}^{2} \widehat{\widehat{Q}}_{n}(t) \bar{P}_{n}(t)\right) \\
= & \varepsilon_{n+1}^{-1}\left(E+\varepsilon_{n} P_{n}(t)\right)^{-1}\left(\varepsilon_{n} \widehat{Q}_{n}^{* *}(t)+\varepsilon_{n}^{2} \widehat{Q}_{n}(t) P_{n}(t)\right) \\
= & \varepsilon_{n}^{-(1+\iota)}\left(E+\varepsilon_{n} P_{n}(t)\right)^{-1}\left(\varepsilon_{n} Q_{n}^{* *}(t)+D_{x} h_{n}^{* *}\left(\underline{x_{n}}(t), t\right)\right) \\
& +\varepsilon_{n}^{-\iota}\left(E+\varepsilon_{n} P_{n}(t)\right)^{-1} \\
& \left(\varepsilon_{n} Q_{n}(t)-\varepsilon_{n} \bar{Q}_{n}(t)+D_{x} h_{n}\left(\underline{x_{n}}(t), t\right)-\bar{D}_{x} h_{n}\left(\underline{x_{n}}(t), t\right)\right) P_{n}(t), \\
& \left(E+\varepsilon_{n} P_{n}(t)\right)^{-1} \widehat{g}_{n}(t) \\
g_{n+1}(t)=(E+ & \left.\varepsilon_{n} P_{n}(t)\right)^{-1} h_{n}\left(\underline{x_{n}}(t), t\right) \varepsilon_{n}^{-(1+\iota)}+\left(E+\varepsilon_{n} P_{n}(t)\right)^{-1} Q_{n}(t) \underline{x_{n}}(t) \varepsilon_{n}^{-\iota},
\end{aligned}
$$




$$
\begin{aligned}
h_{n+1}(t)= & \left(E+\varepsilon_{n} P_{n}(t)\right)^{-1} \widehat{h}_{n}\left(\left(E+\varepsilon_{n} P_{n}(t)\right) x_{n+1}(t), t\right) \\
= & \left(E+\varepsilon_{n} P_{n}(t)\right)^{-1}\left(h_{n}\left(\underline{x_{n}}(t)+\left(E+\varepsilon_{n} P_{n}(t)\right) x_{n+1}, t\right)\right. \\
& \left.\left.\left.-h_{n} \underline{x_{n}}(t), t\right)-D_{x} h_{n} \underline{x_{n}}(t), t\right)\left(E+\varepsilon_{n} P_{n}(t)\right) x_{n+1}\right),
\end{aligned}
$$

where ${\overline{Q_{n}}}_{n}(t)=\overline{\widehat{Q}_{n}^{* *}}(t)=0$. So, by the change of variables $x_{n}(t)=(E+$ $\left.\varepsilon_{n} P_{n}(t)\right) x_{n+1}(t)+\underline{x_{n}}(t)$, Lemma 2.3 and Lemma 2.4, we can transform $(E)_{n}$ to $(E)_{n+1}$.

In the following, we will prove the terms of $(E)_{n+1}$ is bounded by the terms of $(E)_{n}$, and we will use $L_{1, n}, L_{2, n}$ denote the values of $L_{1}, L_{2}$ which is introduced in Lemmas 2.1 and 2.2, also $L_{2, n}^{\prime}$ in place of $L_{2}^{\prime}$. Moreover, as in [9], we use the symbol $K_{n}$ to be the bound of the second derivative of $h_{n}$ and by the same method in [9], we can find $\left\{K_{n}\right\}$ is convergent, we omit the proof.

First we note that a fact $\varepsilon_{n}\left\|P_{n}\right\|^{\Theta_{n+1} \times \mathcal{O}_{n+1}} \leq \frac{1}{2}$ which will be proved in the below, then for $\varepsilon_{n}$ small enough, we will find

$$
\left\|Q_{n+1}\right\|^{\Theta_{n+1} \times \mathcal{O}_{n+1}} \leq 1, \quad\left\|g_{n+1}\right\|^{\Theta_{n+1} \times \mathcal{O}_{n+1}} \leq 1 .
$$

In fact, from Lemmas 2.3 and 2.4, we have

$$
\begin{aligned}
\left\|Q_{n+1}\right\|^{\Theta} \|_{n+1} \times \mathcal{O}_{n+1} & \leq 2 \varepsilon_{n}^{1-\iota} \frac{\left(L_{2, n}^{\prime}+\left\|P_{n}\right\|^{\Theta}{ }^{\Theta+1} \times \mathcal{O}_{n+1}\right)}{1-\varepsilon_{n}\left\|P_{n}\right\|^{\Theta}{ }+1 \times \mathcal{O}_{n+1}}\left\|\widehat{Q}_{n}\right\|^{\Theta\left(\sigma_{n}\right) \times U_{n}} \\
& \leq 4 \varepsilon_{n}^{1-\iota}\left(L_{2, n}^{\prime}+L_{2, n}\left\|\widehat{Q}_{n}\right\| \Theta\left(\sigma_{n}\right) \times U_{n}\right)\left\|\widehat{Q}_{n}\right\|^{\Theta\left(\sigma_{n}\right) \times U_{n}} \\
& \leq 8 L_{1, n}\left(1+K_{n} L_{1, n}\right)\left(3+2 K_{n} L_{1, n}\right) \varepsilon_{n}^{1-\iota}
\end{aligned}
$$

since $L_{1, n}>\max \left\{L_{2, n}, L_{2, n}^{\prime}\right\}$ and

$$
\left\|\widehat{Q}_{n}\right\|^{\Theta\left(\sigma_{n}\right) \times U_{n}}=2\left(\left\|Q_{n}\right\|^{\Theta_{n} \times \mathcal{O}_{n}}+K_{n} L_{1, n}\|g\|^{\Theta_{n} \times \mathcal{O}_{n}}\right),
$$

and $K_{n} \leq\left(\frac{9}{2}\right)^{n} K_{0}$ is the bound of the second derivative of $h_{n}$. We can assume $\gamma \leq 1$, then $L_{1, n}>1$, there is

$$
\left\|Q_{n+1}\right\|^{\Theta_{n+1} \times \mathcal{O}_{n+1}} \leq 24 L_{1, n}^{3}\left(1+K_{n}\right)^{2} \varepsilon_{n}^{1-\iota} \leq 1 .
$$

From Lemmas 2.3 and 2.4 now bound the norm of $g_{n+1}$ as

$$
\begin{aligned}
\left\|g_{n+1}\right\|^{\Theta_{n+1} \times \mathcal{O}_{n+1}} & \leq 2\left\|\widehat{g}_{n}\right\|^{\Theta\left(\sigma_{n}\right) \times U_{n}} \\
& \leq\left(K_{n} L_{1, n}^{2}\left\|g_{n}\right\|^{\Theta_{n} \times \mathcal{O}_{n}}+2 L_{1, n}\left\|Q_{n}\right\|^{\Theta_{n} \times \mathcal{O}_{n}}\left\|g_{n}\right\|^{\Theta_{n} \times \mathcal{O}_{n}}\right) \varepsilon_{n}^{1-\iota} \\
& \leq\left(K_{n}+2\right) L_{1, n}^{2} \varepsilon_{n}^{1-\iota} \leq 1 .
\end{aligned}
$$

Thus, if $\varepsilon$ is a sufficiently small constant, we have

$$
\lim _{n \rightarrow \infty} \varepsilon_{n}\left\|Q_{n}\right\|^{\Theta_{n} \times \mathcal{O}_{n}}=\lim _{n \rightarrow \infty} \varepsilon_{n}\left\|g_{n}\right\|^{\Theta_{n} \times \mathcal{O}_{n}}=0 .
$$

Now we bound $\left\|P_{n}\right\|^{\Theta_{n+1} \times \mathcal{O}_{n+1}}$. From Lemmas 2.3 and 2.4, we have

$$
\left\|P_{n}\right\|^{\Theta_{n+1} \times \mathcal{O}_{n+1}} \leq 2 L_{2, n}\left(\left\|Q_{n}\right\|^{\Theta_{n} \times \mathcal{O}_{n}}+K_{n} L_{1, n}\left\|g_{n}\right\|^{\Theta_{n} \times \mathcal{O}_{n}}\right)
$$




$$
\leq 4\left(\frac{9}{2}\right)^{n} L_{1, n} L_{2, n} K_{0}
$$

and $\lim _{n \rightarrow \infty} \varepsilon_{n}\left\|P_{n}\right\|^{\Theta_{n+1} \times \mathcal{O}_{n+1}}=0$, for $\varepsilon$ small enough, thus we do not mind to take $\varepsilon_{n}\left\|P_{n}\right\|^{\Theta+1} \times \mathcal{O}_{n+1}<\frac{1}{2}$. The bound of $\left\|\underline{x_{n}}\right\|^{\Theta\left(\sigma_{n}\right) \times U_{n}}$ is

$$
\left\|\underline{x_{n}}\right\|^{\Theta\left(\sigma_{n}\right) \times U_{n}} \leq \varepsilon_{n} L_{1, n}\left\|g_{n}\right\|^{\Theta_{n} \times \mathcal{O}_{n}}<\varepsilon_{n} L_{1, n} .
$$

Now we will show that if $\varepsilon$ is small enough, the limit radius $\kappa_{n}$ of the ball where $h_{n}$ analytic with respect to $x$ is positive. Then from Lemma 2.4 and Lemma 2.3 we have

$$
\kappa_{n+1}=\frac{1}{1+\varepsilon_{n}|| P_{n} \|^{\Theta}{ }_{n+1} \times \mathcal{O}_{n+1}} \kappa_{n}-\frac{\left\|x_{n}\right\|^{\Theta\left(\sigma_{n}\right) \times U_{n}}}{1+\varepsilon_{n}|| P_{n} \|^{\Theta}{ }_{n+1} \times \mathcal{O}_{n+1}},
$$

like [9] we define $a_{n}=\frac{1}{1+\varepsilon_{n}\left\|P_{n}\right\|^{\Theta}{ }_{n+1} \times \mathcal{O}_{n+1}}, \quad b_{n}=\frac{\left\|x_{n}\right\|^{\Theta\left(\sigma_{n}\right) \times U_{n}}}{1+\varepsilon_{n}\left\|P_{n}\right\|^{\Theta_{n+1} \times \mathcal{O}_{n+1}}}$, and when $\varepsilon$ is small enough, by Lemma 6.5 it is easy to find $\prod_{n=0}^{\infty} a_{n}$ and $\sum_{n=0}^{\infty} b_{n}$ are convergent, so $\kappa_{\infty} \geq a \kappa_{0}-b>0$.

Now let us bound $\left\|\bar{A}_{n}\right\|$ as

$$
\begin{aligned}
\left\|A_{n+1}\right\| \leq & \left\|\widehat{A}_{n}\right\|+\varepsilon_{n}^{2} \frac{\left(L_{2, n}^{\prime}+\left\|P_{n}\right\|^{\Theta_{n+1} \times \mathcal{O}_{n+1}}\right)}{1-\varepsilon_{n}\left\|P_{n}\right\|^{\Theta_{n+1} \times \mathcal{O}_{n+1}}}\left\|\widehat{Q}_{n}\right\|^{\Theta\left(\sigma_{n}\right) \times U_{n}} \\
\leq & \left\|A_{n}\right\|+\varepsilon_{n}\left(\left\|Q_{n}\right\|^{\Theta_{n} \times \mathcal{O}_{n}}+K_{n} L_{1, n}\left\|g_{n}\right\|^{\Theta} \times \mathcal{O}_{n}\right) \\
& +\varepsilon_{n}^{2} \frac{\left(L_{2, n}^{\prime}+\left\|P_{n}\right\|^{\Theta_{n+1} \times \mathcal{O}_{n+1}}\right)}{1-\varepsilon_{n}\left\|P_{n}\right\|^{\Theta} \widehat{\Theta}_{n+1} \times \mathcal{O}_{n+1}}\left\|\widehat{Q}_{n}\right\|^{\Theta\left(\sigma_{n}\right) \times U_{n}} \\
\leq & \left\|A_{n}\right\|+\varepsilon_{n}\left(1+\left(\frac{9}{2}\right)^{n} K_{0} L_{1, n}\right) \\
& +4 \varepsilon_{n}^{2}\left(1+\left(\frac{9}{2}\right)^{n} K_{0} L_{1, n}\right)\left(L_{2, n}^{\prime}+4\left(\frac{9}{2}\right)^{n} L_{1, n} L_{2, n} K_{0}\right) \\
\leq & \left\|A_{n}\right\|+\varsigma_{n},
\end{aligned}
$$

where $\varsigma_{n} \leq \varepsilon_{n} C^{\prime}$ for a suitable $C^{\prime}$, when $\varepsilon$ is small enough, we have $\sum_{n=1}^{\infty} \varsigma_{n}$ convergent, so we can ensure that the matrices $A_{n} \rightarrow B$, when $n \rightarrow \infty$.

\section{Proof of Theorem 1.1}

We remark that the system (1.3) satisfies $\left(E_{\nu}\right),(H 1)_{\nu}$ and $(H 2)_{\nu}$ with $\nu=0$, the iterative procedure in Lemma 3.1 can run repeatedly. So, there exists a sequence of transformation $x_{n}=\left(E+\varepsilon_{n} P_{n}(t, \omega)\right) x_{n+1}+\underline{x_{n}}, n=0,1,2, \ldots$, such that $P_{n}(t)$ are analytic in the domains $\Theta_{n+1} \times \mathcal{O}_{n+1}$. Let

$$
\Theta_{\infty} \times \mathcal{O}_{\infty}=\bigcap_{n=1}^{\infty} \Theta_{n} \times \mathcal{O}_{n}
$$

Then, all the $P_{n}, n=1,2, \ldots$, are well defined in the domain $\Theta_{\infty} \times \mathcal{O}_{\infty}$, and we have

$$
\begin{aligned}
x_{n+1} & =\Phi_{n}^{-1}(t) x_{n}=\Phi_{n}^{-1}(t) \circ \Phi_{n-1}^{-1}(t) x_{n-1} \\
& =\cdots
\end{aligned}
$$




$$
=\Phi_{n}^{-1}(t) \circ \Phi_{n-1}^{-1}(t) \circ \cdots \circ \Phi_{0}^{-1}(t) x_{0},
$$

i.e.,

$$
\begin{aligned}
x_{n+1}= & \left(E+\varepsilon_{n} P_{n}\right)^{-1} x_{n}-\left(E+\varepsilon_{n} P_{n}\right)^{-1} \underline{x_{n}} \\
= & \left(E+\varepsilon_{n} P_{n}\right)^{-1} \circ\left(\left(E+\varepsilon_{n-1} P_{n-1}\right)^{-1} x_{n-1}\right. \\
& \left.-\left(E+\varepsilon_{n-1} P_{n-1}\right)^{-1} \underline{x_{n-1}}\right)-\left(E+\varepsilon_{n} P_{n}\right)^{-1} \underline{x_{n}} \\
= & \left(E+\varepsilon_{n} P_{n}\right)^{-1} \circ\left(E+\varepsilon_{n-1} P_{n-1}\right)^{-1} x_{n-1} \\
& -\left(E+\varepsilon_{n} P_{n}\right)^{-1} \circ\left(E+\varepsilon_{n-1} P_{n-1}\right)^{-1} \underline{x_{n-1}} \\
& -\left(E+\varepsilon_{n} P_{n}\right)^{-1} \underline{x_{n}} \\
= & \cdots \\
= & \left(E+\varepsilon_{n} P_{n}\right)^{-1} \circ\left(E+\varepsilon_{n-1} P_{n-1}\right)^{-1} \circ \cdots \circ\left(E+\varepsilon_{0} P_{0}\right)^{-1} x_{0} \\
& -\sum_{i=0}^{n}\left(E+\varepsilon_{n} P_{n}\right)^{-1} \circ \cdots \circ\left(E+\varepsilon_{i} P_{i}\right)^{-1} \underline{x_{i}},
\end{aligned}
$$

where $\underline{x_{i}}$ is a solution of the equation as in Lemma 2.1.

We $\overline{\text { set }}$

Note that

$$
\Psi_{n}(t)=\Phi_{n}^{-1}(t) \circ \Phi_{n-1}^{-1}(t) \circ \cdots \circ \Phi_{0}^{-1}(t) .
$$

$$
\left\|\underline{\mid x_{n}}\right\|^{\Theta \infty \times U_{\infty}} \leq\left\|\left.\underline{x_{n}}\right|^{\Theta\left(\sigma_{n}\right) \times U_{n}}<\varepsilon_{n} L_{1, n}, \quad \lim _{n \rightarrow \infty} \varepsilon_{n}\right\| P_{n} \|^{\Theta_{n+1} \times \mathcal{O}_{n+1}}=0,
$$

then

$$
\begin{aligned}
& \left\|\Psi_{n+1}(t)-\Psi_{n}(t)\right\|^{\Theta_{\infty} \times U_{\infty}} \\
= & \left\|\Phi_{n+1}^{-1}(t) \circ \Psi_{n}(t)-\Psi_{n}(t)\right\|^{\Theta_{\infty} \times U_{\infty}} \\
\leq & \left\|\Phi_{n+1}^{-1}(t)-E\right\|^{\Theta_{\infty} \times U_{\infty}}\left\|\Psi_{n}(t)\right\|^{\Theta_{\infty} \times U_{\infty}} \\
\leq & \left(\left\|\left(E+\varepsilon_{n+1} P_{n+1}\right)^{-1}-E\right\| \|_{\infty}^{\Theta_{\infty} \times U_{\infty}}\right. \\
& \left.+\left\|\left(E+\varepsilon_{n+1} P_{n+1}\right)^{-1} \underline{x_{n+1}}\right\|^{\Theta_{\infty} \times U_{\infty}}\right)\left\|\Psi_{n}(t)\right\|^{\Theta_{\infty} \times U_{\infty}} \\
\leq & \left(\frac{\varepsilon_{n+1}\left\|P_{n+1}\right\| \|_{n+2} \times U_{n+2}}{1-\varepsilon_{n+1} \| P_{n+1}||^{\Theta_{n+2} \times U_{n+2}}}+\frac{\varepsilon_{n+1} L_{1, n+1}}{1-\varepsilon_{n+1}\left\|P_{n+1}\right\|^{\Theta_{n+2} \times U_{n+2}}}\right)\left\|\Psi_{n}(t)\right\|_{\infty}^{\Theta_{\infty} \times U_{\infty}} \\
\leq & \left(2 \varepsilon_{n+1}\left\|P_{n+1}\right\|^{\Theta_{n+2} \times U_{n+2}}+2 \varepsilon_{n+1} L_{1, n+1}\right)\left\|\Psi_{n}(t)\right\|^{\Theta_{\infty} \times U_{\infty}},
\end{aligned}
$$

it is easy to find that

$$
\left\|\Psi_{n}(t)\right\|^{\Theta_{\infty} \times U_{\infty}}<+\infty
$$

so $\left\{\Psi_{n}(t)\right\}$ is convergent on $\Theta_{\infty} \times U_{\infty}$, where we use $\left.\varepsilon_{n+1}\left\|P_{n+1}\right\|\right|^{\Theta_{n+2} \times \mathcal{O}_{n+2}}<\frac{1}{2}$ as in Lemma 3.1. We see that $\Psi(t)=\lim _{n \rightarrow \infty} \Psi_{n}(t)$ is well defined, let

$$
y=\Psi(t) x .
$$


Then, the transformation $y=\Psi(t) x$ change (1.3) into

$$
\dot{y}=A_{\infty}(\varepsilon) y+h_{\infty}(y, t, \varepsilon)
$$

This completes the proof of Theorem 1.1.

\section{Measure lemma of the allowed frequencies set}

In this section, we bound the measure of the resonances and in the proving, $C$ is positive constants.

Lemma 5.1. Let $\tau=\tau_{1}+d^{2}-1$ and

$$
\begin{gathered}
G^{*}(\omega)=\sqrt{-1}(k, \omega) E_{d^{2}}-E_{d} \otimes A_{n}+A_{n}^{\top} \otimes E_{d}, \\
G^{* *}(\omega)=\sqrt{-1}(k, \omega) E_{d}-A_{n}(\omega) .
\end{gathered}
$$

Then, for $\omega \in \mathcal{O}_{n}$ and $0<|k| \leq M_{n}$, the inverse of $G^{*}(\omega)$ and $G^{* *}(\omega)$ exist, moreover, they are analytic in the domain $\mathcal{O}_{n}$ with

$$
\left(G^{*}\right)^{-1}(\omega) \leq C \frac{|k|^{\tau}}{\gamma_{n}}, \quad\left(G^{* *}\right)^{-1}(\omega) \leq C \frac{|k|^{\tau}}{\gamma_{n}},
$$

respectively.

Proof. This proof like the Lemma 3.1 in [16], we omit it.

Lemma 5.2. Let $R=d^{2}(d+1)^{2}\left(\text { diam } \Pi_{0}\right)^{r-1}$. Then the Lebesgue measure of $\mathcal{R}_{k}(n)$ satisfies

$$
\operatorname{Meas} \mathcal{R}_{k}(n) \leq \frac{2}{n^{2}} \frac{R \gamma^{\frac{1}{d^{2}}}}{|k|^{r+1}}
$$

Proof. From Lemma 2.4 and Lemma 2.3 we have

$$
A_{n+1}(\omega)=A_{n}(\omega)+\varepsilon_{n} \bar{Q}_{n}(\omega)+\overline{D_{x} h\left(\underline{x}_{n}(t, \omega), t\right)} .
$$

First, we know that $q_{s}=\varepsilon_{s+1}^{\frac{1}{4 d^{2}}}$, let $q_{1, s}=\frac{5}{6} q_{s}+\frac{1}{6} q_{s+1}, q_{2, s}=\frac{11}{12} q_{s}+\frac{1}{12} q_{s+1}$, and $\mathcal{O}_{i, s}$ are the $q_{i, s}$-neighborhood of $\Pi_{s}$ respectively for $i=1,2$, moreover, $\rho_{1, s}=\frac{5}{6} \rho_{s}+\frac{1}{6} \rho_{s+1}, \rho_{2, s}=\frac{11}{12} \rho_{s}+\frac{1}{12} \rho_{s+1}$ are the size of the analyticity domain in the angular variables. Obviously, $\mathcal{O}_{s+1} \subset \mathcal{O}_{1, s} \subset \mathcal{O}_{2, s} \subset \mathcal{O}_{s}$ and $\operatorname{dist}\left(\partial \mathcal{O}_{1, s}, \partial \mathcal{O}_{s}\right)>\operatorname{dist}\left(\partial \mathcal{O}_{2, s}, \partial \mathcal{O}_{s}\right)=\operatorname{dist}\left(\partial \mathcal{O}_{1, s}, \partial \mathcal{O}_{2, s}\right)>\frac{1}{24} q_{s}$, and $\rho_{s+1}<\rho_{1, s}<\rho_{2, s}<\rho_{s}$. Recall that $\left\|Q_{s}\right\|^{\Theta_{s} \times \mathcal{O}_{s}} \leq 1$ and $\left\|g_{s}\right\|^{\Theta_{s} \times \mathcal{O}_{s}} \leq 1$, then for $1 \leq l \leq d^{2}$ and $0 \leq s \leq n$,

$$
\begin{gathered}
\varepsilon_{s}\left\|\partial_{\omega}^{l} \bar{Q}_{s}(\omega)\right\|^{\Theta_{1, s} \times \mathcal{O}_{1, s}} \leq \varepsilon_{s} \frac{24^{l}}{q_{s}^{l}}\left\|\bar{Q}_{s}(\omega)\right\|^{\Theta_{s} \times \mathcal{O}_{s}} \leq \varepsilon_{s} \frac{24^{l}}{q_{s}^{l}}\left\|Q_{s}(\omega)\right\|^{\Theta_{s} \times \mathcal{O}_{s}} \leq \varepsilon_{s}^{\frac{1}{2}}, \\
\left\|\partial_{\omega}^{l} \overline{D_{x} h\left(\underline{x}_{s}(t, \omega), t\right)}\right\|^{\Theta_{1, s} \times \mathcal{O}_{1, s}} \leq \frac{24^{l}}{q_{s}^{l}}\left\|\overline{D_{x} h\left(\underline{x}_{s}(t, \omega), t\right)}\right\|^{\Theta_{2, s} \times \mathcal{O}_{2, s}}
\end{gathered}
$$




$$
\begin{aligned}
& \leq \frac{24^{l}}{q_{s}^{l}}\left\|D_{x} h\left(\underline{x}_{s}(t, \omega), t\right)\right\|^{\Theta_{2, s} \times \mathcal{O}_{2, s}} \\
& \leq \frac{24^{l}}{q_{s}^{l}} K_{s}\left\|\underline{x}_{s}(t, \omega)\right\| \|^{\Theta_{2, s} \times \mathcal{O}_{2, s}} \\
& \leq \varepsilon_{s} \frac{24^{l}}{q_{s}^{l}} K_{s} \widetilde{L}_{1, s}\left\|g_{s}(\omega)\right\|^{\Theta_{s} \times \mathcal{O}_{s}} \leq \varepsilon_{s}^{\frac{1}{2}} .
\end{aligned}
$$

Now from (5.6) and (5.7), the norm boundedness of every terms in (5.5) and recursiveness, for a sufficiently small $\varepsilon$, we have

$$
\left\|\partial_{\omega}^{l} A_{n+1}(\omega)\right\|^{\Theta_{1, n} \times \mathcal{O}_{1, n}} \leq \varepsilon^{\frac{1}{2}} .
$$

Let $B(\omega)=-E_{d} \otimes A_{n}(\omega)+A_{n}^{\top}(\omega) \otimes E_{d}=\left(b_{i j}\right)$. Then

$$
\left\|\partial_{\omega}^{l} B(\omega)\right\|^{\Theta_{1, n-1} \times \mathcal{O}_{1, n-1}} \leq \varepsilon^{\frac{1}{2}},
$$

obviously, for $1 \leq l \leq d^{2}$ and $\omega \in \mathcal{O}_{1, n-1}$,

$$
\left|\partial_{\omega_{1}}^{l} b_{i j}\right| \leq\left\|\partial_{\omega}^{l} B(\omega)\right\|^{\Theta_{1, n-1} \times \mathcal{O}_{1, n-1}} \leq \varepsilon^{\frac{1}{2}} .
$$

Set $\mathcal{T}(\omega)=\left|\sqrt{-1}(k, \omega) E_{d^{2}}+B(\omega)\right|_{e}$. Then

$$
\mathcal{T}(\omega)=\sqrt{-1}^{d^{2}}(k, \omega)^{d^{2}}+\sum_{1 \leq \nu \leq d^{2}-1} \chi_{\nu}(\omega)(k, \omega)^{d^{2}-\nu}
$$

where

$$
\chi_{\nu}(\omega)=\sum_{1 \leq j_{i} \leq d^{2}} \sigma_{j_{1} \cdots j_{\nu}} b_{1 j_{1}} \cdots b_{\nu j_{\nu}} \text { and } \sigma_{j_{1} \cdots j_{\nu}} \in\{-1,+1,-\sqrt{-1},+\sqrt{-1}\} .
$$

For $\omega \in \mathcal{O}_{1, n-1}, 1 \leq l \leq d^{2}$, by $(5.8)$,

$$
\begin{aligned}
\left|\frac{d^{l}}{d \omega_{1}^{l}}\left(b_{1 j_{1}} \cdots b_{l j_{\nu}}\right)\right| & \leq\left|\sum_{l_{1}+\cdots+l_{\nu}=l}\left(\frac{d^{l_{1}}}{d \omega_{1}^{l_{1}}} b_{1 j_{1}}\right) \cdots\left(\frac{d^{l_{\nu}}}{d \omega_{1}^{l_{\nu}}} b_{1 j_{\nu}}\right)\right| \\
& \leq \varepsilon^{\frac{l}{2}} \sum_{l_{1}+\cdots+l_{\nu}=l} 1 \\
& \leq 2^{l} \varepsilon^{\frac{l}{2}}
\end{aligned}
$$

and

$$
\left|\frac{d^{l}}{d \omega_{1}^{l}} \chi_{\nu}(\omega)\right| \leq\left(\begin{array}{c}
d^{2} \\
\nu
\end{array}\right) 2^{l} \varepsilon^{\frac{l}{2}}
$$

Now, without loss of generality, we can assume that $|k|=\left|k_{1}\right|+\cdots+\left|k_{r}\right| \leq r\left|k_{1}\right|$. Then, for every $\omega \in \mathcal{O}_{1, n-1}$,

$$
\left|\frac{d^{d^{2}}}{d \omega_{1}^{d^{2}}} \sum_{1 \leq \nu \leq d^{2}-1} \chi_{\nu}(\omega)(k, \omega)^{d^{2}-\nu}\right|
$$




$$
\begin{aligned}
& \leq \sum_{1 \leq \nu \leq d^{2}-1}\left|\frac{d^{d^{2}}}{d \omega_{1}^{d^{2}}}\left(\chi_{\nu}(\omega)(k, \omega)^{d^{2}-\nu}\right)\right| \\
& \leq \sum_{1 \leq \nu \leq d^{2}-1} \sum_{1 \leq l \leq d^{2}}\left(\begin{array}{c}
d^{2} \\
l
\end{array}\right)\left|\frac{d^{l}}{d \omega_{1}^{l}} \chi_{\nu}(\omega)\right|\left|\frac{d^{d^{2}-l}}{d \omega_{1}^{d^{2}-l}}(k, \omega)^{d^{2}-\nu}\right| \\
& \leq \sum_{1 \leq \nu \leq d^{2}-1} \sum_{1 \leq l \leq d^{2}}\left(\begin{array}{c}
d^{2} \\
\nu
\end{array}\right)\left(\begin{array}{c}
d^{2} \\
l
\end{array}\right) 2^{l} \varepsilon^{\frac{l}{2}}\left|k_{1}\right|^{d^{2}-l}|(k, \omega)|^{l-\nu} d^{2} ! \\
& \leq \varepsilon^{\frac{1}{2}} C_{1}\left|k_{1}\right|^{d^{2}-1} d^{2} !,
\end{aligned}
$$

where $C_{1}$ is some constant which depends only on $d, r$ and on the maximum of $|\omega|$ in $\Pi_{0}$. Obviously,

$$
\frac{d^{d^{2}}}{d \omega_{1}^{d^{2}}}(k, \omega)^{d^{2}}=d^{2} !\left|k_{1}\right|^{d^{2}}
$$

moreover, in $\mathcal{O}_{n-1}^{1}$, for $\varepsilon$ small enough so that $C_{1} \varepsilon^{\frac{1}{2}}<\frac{1}{2}$, by (5.12) and (5.13) we have

$$
\left|\frac{d^{d^{2}}}{d \omega_{1}^{d^{2}}} \mathcal{T}(\omega)\right| \geq d^{2} !\left|k_{1}\right|^{d^{2}}\left(1-\varepsilon^{\frac{1}{2}} C_{1}\left|k_{1}\right|^{-1}\right) \geq \frac{1}{2} d^{2} !\left|k_{1}\right|^{d^{2}} .
$$

Thus, from (5.14) and Lemma 6.3, we get

$$
\operatorname{Meas}_{k}^{*}(n) \leq d^{2}(d+1)^{2}\left(\frac{\gamma_{n}}{|k|^{\tau_{1}}}\right)^{\frac{1}{d^{2}}}\left(\operatorname{diam} \Pi_{0}\right)^{r-1} \leq \frac{1}{n^{2}} \frac{R \gamma^{\frac{1}{d^{2}}}}{|k|^{r+1}} .
$$

For the $\operatorname{Meas}_{k}^{* *}(n)$, we also have

$$
\operatorname{Meas}_{k}^{* *}(n) \leq \frac{1}{n^{2}} \frac{R \gamma^{\frac{1}{d^{2}}}}{|k|^{r+1}} .
$$

It means

$$
\operatorname{Meas}_{k}(n) \leq \operatorname{Meas}_{k}^{*}(n)+\operatorname{Meas}_{k}^{* *}(n) \leq \frac{2}{n^{2}} \frac{R \gamma^{\frac{1}{d^{2}}}}{|k|^{r+1}} .
$$

We can find the nest sequence of closed sets

$$
\Omega_{0}=\Pi_{0} \supset \Pi_{1} \supset \cdots \supset \Pi_{n} \supset \cdots
$$

is defined inductively. Moreover, we have the following lemma.

Lemma 5.3. Let $\Pi_{\infty}=\bigcap_{n=0}^{\infty} \Pi_{m}$. Then the Lebesgue measure of $\Pi_{\infty}$ satisfies

$$
\operatorname{Meas} \Pi_{\infty}=\left(\operatorname{Meas} \Pi_{0}\right)\left(1-O\left(\gamma^{\frac{1}{d^{2}}}\right)\right) .
$$




\section{Appendix}

Lemma 6.1. For $\delta>0$ and $\nu>0$, the following inequality holds true:

$$
\sum_{k \in \mathbb{Z}^{N}}|k|^{\nu} e^{-2|k| \delta} \leq\left(\frac{\nu}{e}\right)^{\nu} \frac{(1+e)^{N}}{\delta^{\nu+N}} .
$$

This lemma can be found in [1].

The following lemma can be found in many books on matrix theory; for example in [10].

Lemma 6.2. Let $A, B$ and $C$ be $l \times l, m \times m$ and $l \times m$ matrixes, respectively; and let $X$ be an $l \times m$ unknown matrix. Then the matrix equation

$$
A X+X B=C
$$

is solvable if and only if the vector equation

$$
\left(E_{m} \otimes A+B^{\top} \otimes E_{l}\right) X^{\prime}=C^{\prime}
$$

is solvable, where $X^{\prime}=\left(X_{1}^{\top}, \ldots, X_{m}^{\top}\right)^{\top}, C^{\prime}=\left(C_{1}^{\top}, \ldots, C_{m}^{\top}\right)^{\top}$ if we write $X=$ $\left(X_{1}, \ldots, X_{m}\right)$ and $C=\left(C_{1}, \ldots, C_{m}\right)$. Moreover,

$$
\|X\| \leq\left\|\left(E_{m} \otimes A+B^{\top} \otimes E_{l}\right)^{-1}\right\|\|C\|
$$

if the inverse exists.

Lemma 6.3 ([15], Lemma 4.1). Let $\Delta$ be an interval in $\mathbb{R}^{1}$ and $\bar{\Delta}$ its closure. Suppose that $\psi: \bar{\Delta} \rightarrow \mathbb{C}$ is $k$ times continuously differentiable. Let $\Delta_{j}=\{x \in$ $\bar{\Delta}:|\psi(x)| \leq j\}, j>0$. If, for some constant $\nu>0,\left|\frac{d^{k} \psi(x)}{d x^{k}}\right| \geq \nu$ for any $x \in \Delta$, then MeasI $_{j} \leq c j^{\frac{1}{k}}$ where $c=2\left(2+3+\cdots+k+\nu^{-1}\right)$.

Lemma 6.4 ([9], Lemma 2). Let $h: U \subset \mathbb{R}^{d} \rightarrow \mathbb{R}^{d}$ be a function of class $\mathcal{C}^{2}$ on a ball $B_{\kappa}(0)$ that satisfies $h(0)=0, D_{x} h(0)=0$, and $\left\|D_{x x} h(x)\right\| \leq K$, where $x \in B_{\kappa}(0)$. Then $\|h(x)\| \leq \frac{K}{2}\|x\|^{2}$ and $\left\|D_{x} h(x)\right\| \leq K\|x\|$.

Lemma 6.5 ([9], Lemma 10). Let $\left\{a_{n}\right\}$ be a sequence of positive real numbers that satisfies $a_{n} \in(0,1], \prod_{n=0}^{\infty} a_{n}=a \in(0,1]$. Let $\left\{b_{n}\right\}$ be another sequence of positive real numbers that satisfies $\sum_{n=0}^{\infty} b_{n}=b<+\infty$. We define a new sequence $\left\{\kappa_{n}\right\}$ is $\kappa_{n+1}=a_{n} \kappa_{n}-b_{n}$. Then $\kappa_{n} \rightarrow \kappa_{\infty}, n \rightarrow \infty$, and $\kappa_{\infty} \geq a \kappa_{0}-b$.

Acknowledgements. The author is very grateful to the Professor Jianguo Si (School of mathematics, Shandong University in China) for valuable and inspiring suggestions.

\section{References}

[1] N. N. Bogoljubov, Ju. A. Mitropoliski, and A. M. Samoilenko, Methods of Accelerated Convergence in Nonlinear Mechanics, Springer-Verlag, New York, 1976.

[2] W. A. Coppel, Pseudo-autonomous linear system, Bull. Austral. Math. Soc. 16 (1977), no. $1,61-65$. 
[3] E. I. Dinaburg and Y. G. Sinai, The one dimensional Schrödinger equation with a quasiperiodic potential, Funkcional. Anal. i Priložen. 9 (1975), no. 1, 8-21.

[4] L. H. Eliasson, Floquet solutions for the 1-dimensional quasi-periodic Schrodinger equation, Comm. Math. Phys. 146 (1992), no. 3, 447-482.

[5] H. Her and J. You, Full measure reducibility for generic one-parameter family of quasiperiodic linear systems, J. Dynam. Differential Equations 20 (2008), no. 4, 831-886.

[6] R. A. Johnson and G. R. Sell, Smoothness of spectral subbundles and reducibility of quasiperiodic linear differential systems, J. Differential Equations 41 (1981), no. 2, 262288.

[7] A. Jorba, R. Ramŕez-ros, and J. Villanueva, Effective reducibility of quasi-periodic linear equations close to constant coefficients, SIAM J. Math. Anal. 28 (1997), no. 1, 178-188.

[8] A. Jorba and C. Simó, On the reducibility of linear differential equations with quasiperiodic coefficients, J. Differential Equations 98 (1992), no. 1, 111-124.

[9] _ On quasi-periodic perturbations of elliptic equilibrium points, SIAM J. Math. Anal. 27 (1996), no. 6, 1704-1737.

[10] P. Lancaster, Theory of Matrices, Academic Press, New York, 1969.

[11] J. Moser, Convergent series expansions for quasi-periodic motions, Math. Ann. 169 (1967), 136-176.

[12] J. Moser and J. Pöschel, On the stationary Schrödinger equation with a quasiperiodic potential, Phys. A 124 (1984), no. 1-3, 535-542.

[13] H. Rüssmann, On the one dimensional Schrödinger equation with a quasiperiodic potential, Nonlinear dynamics (Internat. Conf., New York, 1979), pp. 90-107, Ann. New York Acad. Sci., 357, New York Acad. Sci., New York, 1980.

[14] J. Xu and Q. Zheng, On the reducibility of linear differential equations with quasiperiodic coeffficients which are degenerate, Proc. Amer. Math. Soc. 126 (1998), no. 5, 1445-1451.

[15] J. You, Perturbations of lower-dimensional tori for Hamiltonian systems, J. Differential Equations 152 (1999), no. 1, 1-29.

[16] X. P. Yuan and A. Nunes, A note on the reducibility of linear differential equations with quasi-periodic coefficients, Int. J. Math. Math. Sci. 2003 (2003), no. 64, 4071-4083.

SCHOOL OF MATHEMATICS

Chongqing Normal University

Chongqing 401331, P. R. China

E-mail address: houyu19@gmail.com 